EESTI NSV TEADUSTE AKADEEMIA TOIMETISED. X KOIDE

FOOSIKALIS-MATEMAATILISTE JA TEHNILISTE TEADUSTE SEERIA, 1961, NR. 4

ИЗВЕСТИЯ АКАДЕМИИ НАУК ЭСТОНСКОИ ССР. ТОМ Х СЕРИЯ ФИЗИКО-МАТЕМАТИЧЕСКИХ И ТЕХНИЧЕСКИХ НАУК. 1961, N 4

\title{
АВТОМАТИЧЕСКОЕ УПРАВЛЕНИЕ УСТАНОВКИ ТЕРМИЧЕСКОИ ПЕРЕРАБОТКИ СЛАНЦА С ТВЕРДЫМ ТЕПЛОНОСИТЕЛЕМ ПРИ ПОМОЩИ ЭКСТРЕМАЛЬНОГО РЕГУЛЯТОРА
}

\section{Э. КЮННАП,}

\author{
кандидат технических наук
}

Используемые в настоящее время промышленные сланцеперерабатывающие установки - туннельные печи, шахтные генераторы, вращающиеся реторты - имеют теп. лоноситель в виде газа. Они относительно маломощны из-за крупности кусков перерабатываемого сырья и малой интенсивности теплопередачи от греющего газа к перерабатываемому сланцу, и поэтому плохо поддаются автоматическому регулированию. В то же время процесс термической переработки сланца в установке с твердым теплоносителем поддается регулированию сравнительно легче. Благодаря тому, что в качестве сырья здесь нспользуется сланцевая мелочь, а теплоносителем является горячая зола из аэрофонтанной топки, нагревание куска происходит значительно быстрее, чем в названных выше установках, а условия теплопередачи от золы к сырью значительно лучше, чем от газов к сырью. Поэтому установки такого типа мощнее вышеописанных.

Основными конечными продуктами переработки сланца могут быть либо смола, либо высококалорийный газ. На комбинате «Кивиыли» уже работает опытная установка с твердым теплоносителем производительностью 250 т сланца в сутки. Там же начато строительство другой такой же установки мощностью 500 т сланца в сутки. Принципнальная схема установки этого типа приведена на фиг. 1.

В реакторе 1 происходит смешение теплоносителя (золы) с мелким сланцем и осуществляется теплообмен между ними. За счет физического тепла золы совершается процесс термического разложения органической части сланца, Сырой мелкозернистый сланец подается в аэрофонтанную сушилку 5, в которой 3 а счет тепла топочных газов, поступающих из аэрофонтанной топки 2, циклона теплоносителя 3 и зольного циклона 4 , происходит сушка н подогрев топлива до температуры 110 $120^{\circ}$ С. Далее аэросмесь сухого сланца и топочных газов поступает в циклон сухого сланца $\vec{b}$,

Фиг. 1. Принципиальная схема установки термической переработки сланца с твердым теплоносителем: 1 - реактор; 2 аэрофонтанная топка; 3 - циклон теплоносителя: 4 - зольный циклон; 5-сушилка; 6 - щиклон сухого сланца; 7 байпасный клапан.

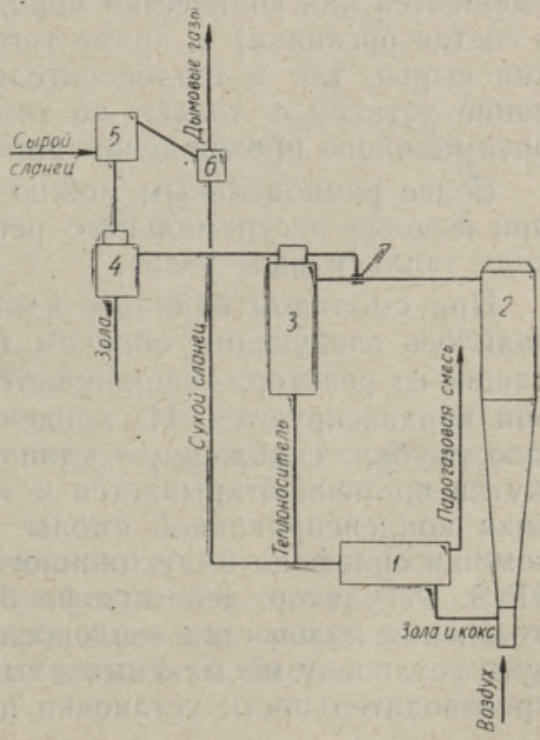


где происходит разделение сухого сланца и топочных газов. Топочные газы выбрасываются в атмосферу, а сухой сланец направляется в реактор, куда поступает теплоноситель - зола из циклона теплоносителя 3. Парогазовая смесь, образующаяся в реакторе вследствие термического разложения сланца, после очистки от пыли направляется в конденсацию, а твердые остатки в виде смеси золы и полукокса, имеюшие теплоту сгорания порядка 250-300 ккал/кг, направляются в аэрофонтанную топку 2. В аэрофонтанной топке происходит дожигание горючего, находящегося в смеси золы ": полукокса, воздухом, подаваемым в нижнюю часть топки. В результате горения обра зуются топочные газы и зола. Поскольку количество золы, выходящей из аэрофон танной топки 2, превышает потребности реактора, то часть ее и газы направляются прямо в зольный циклон 4, где происходит отделенне золы от дымовых газов. Полученная зола может быть использована в качестве строительного материала (роман цемент), а при помощи дымовых газов, как указано выше, происходит сушка сырого сланща в сушнлке 5. Регулирование количества поступающего в реактор теплоносителя производится с помощью байпасного клапана 7.

Если при регулнровании технологического процесса в реакторе пренебречь влияннем на результат регулирования таких факторов, как изменение гранулометрического состава сланца и теплоносителя, измененне теплотворности ссланца и др., то прн поддерживании оптимального теплового режима в реакторе процесс также должен протекать оптимально. В этом случае необходимо только стабилизировать процесс сушки сланца, процесс горения в аэрофонтанной топке и температуру в реакторе. Для облегчения стабилизации теплового режима в реакторе необходимо еще стабилизировать давление паро-газовой смеси в снстеме конденсацин.

Однако такая система регулирования обеспечивает только некоторую стабилиза цию процесса и далеко не соответствует оптимальным условиям его протекания, так как задачей рассмотренного процесса является получение из данного количества и состава сырья, находящегося в рассмотренный промежуток времени в реакторе, либо максимального количества смолы (если установка работает в смоляном варианте), либо максимального количества тепла в виде газа (если установка работает в газовом варианте). Кроме того, еще не точно установлена наиболее выгодная температура в реакторе, которая по разным литературным данным находится в пределах $450-520^{\circ}$, если установка работает в смоляном режиме, и $650-750^{\circ}$ при газовом режиме.

Учитывая, что рабочий состав сланца - величина непостоянная (меняются как количество породы, так и влажность, а в меньшей мере и состав органики) и, кроме того, меняются гранулометрический состав как сырья, так и теплоносителя и условия теплопередачи, регулирование установки только по температуре в реакторе не обеспечивает оптимального прохождения процесса.

Более рациональным можно считать систему управления установкн при помоци экстремального регулятора по выходу смолы (или по выходу тепла в виде газа).

При смоляном варнанте измерение выхода смоляных паров пронзводилось следующим образом (фиг, 2). Часть смоляных паров, выходящих из реактора, направляется в конденсатор (охладитель) КР, где они конденскруются. Из конденсатора смола поступает в измерительную трубку, снабженную клапаном, который в строго равные промежутки времени открывается и закрывается регулятором. Высота столбика конденсированной смолы измерительной трубки измеряется при помощи сильфона индукционного датчика ИД и передается регулятору ЭР-Э. Регулятор, действуя на байпасный клапан теплоносителя путем изменения количества теплоносителя, поступающего в реактор, регулирует установку на максимальный выход смолы. Результаты измерения пронзводительности установки приведены на фиг. 3 , откуда видно, что 
в данном случае оптимальной температурой в реакторе можно считать примерно $500-510^{\circ}$.

Система регулирования при смоляном варианте (см. фиг. 2) состоит, таким образом, из регулятора температуры газов, уходящих из аэрофонтанной топки ЭРТ2-54, регулятора давления смоляных паров

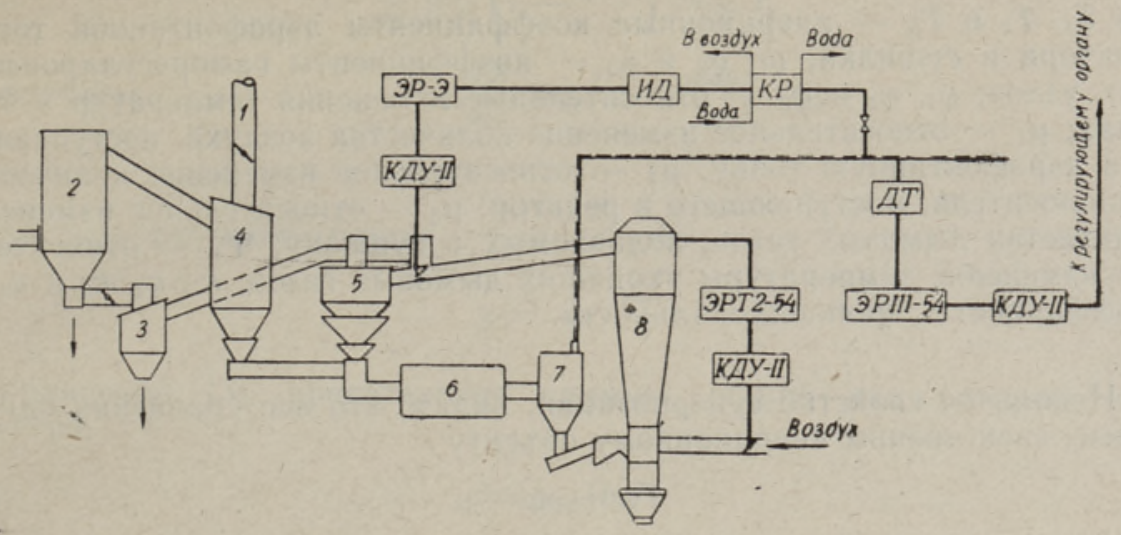

Фиг. 2. Система регулирования.

в системе ЭРІІІ-54 и экстремального регулятора ЭР-Э. При такой схеме регулирования отпадает необходимость учитывать прочие указанные выше компоненты, влияющие на процесс термического разложения. Не нужно при этом учитывать и изменения характеристикн постоянства байпасного клапана и система регулирования получается сравнительно простой.

Bсе указанные регуляторы - электронные, стандартные, системы ВТИ. Исключение составляет лишь последний, экстремальный регулятор, который также является электронным, но разработан и изгоговлен в Институте кибернетики АН ЭССР.

Фиг. 3. Кривые пронзводительности установки при работе в смоляном варианте в зависимости от температуры в реакторе: $A$ условная кривая, $Б$ - усредненная крнвая; точки $C$ - результаты измерения.

Для теоретического исследования автоматическо. го регулирования установки необходимо иметь числовые значения ннерционных коэффициентов его уравне-

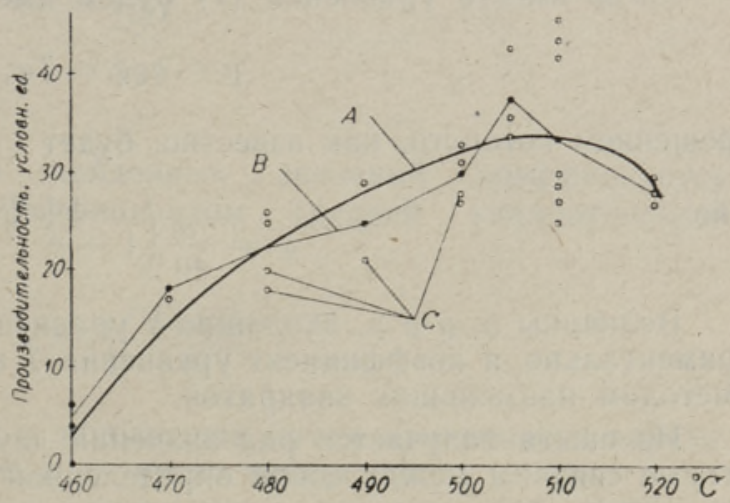
ний. Термическая переработка происходит в реакторе путем смешения золы с сухим сланцем. Зола-теплоноситель подготовляется в аэрофонтанной топке, а сланец в сушилке. Следовательно, необходимо иметь уравнения реактора, сушилки н аэрофонтанной топки. Исходя из тепловых балансов этих узлов, найдем эти уравнения: 


$$
\begin{aligned}
& T_{1} \dot{\varphi}_{1}+\varrho_{1} \varphi_{1}=\mu_{1}-\text { уравнение аэрофонтанной топки } \\
& T_{2} \dot{\varphi_{2}}+\varrho_{2} \varphi_{2}=\mu_{2}+\kappa_{1} \varphi_{1}+\kappa_{2} \varphi_{3}-\text { уравнение реактора } \\
& T_{3} \dot{\varphi}_{3}+\varrho_{3} \varphi_{3}=\mu_{3}+\kappa_{3} \varphi_{1}+\kappa_{4} \Psi_{1}-\text { уравнение сушилки, }
\end{aligned}
$$

где $T_{1}, T_{2}$ и $T_{3}$ - инерционные коэффициенты аэрофонтанной топки, реактора и сушилки; $\varrho_{1}, \varrho_{2}$ и $\varrho_{3}-$ коэффициенты саморегулирования этих узлов; $\varphi_{1}, \varphi_{2}$ и $\varphi_{3}$ - относительные изменения температур в этих узлах; $\mu_{1}$ - относительное изменение количества воздуха, поступающего в аэрофонтанную топку; $\mu_{2}$ - относительное изменение количества теплоносителя, поступающего в реактор; $\mu_{3}$ - относительное изменение количества дымовых газов, подаваемых в сушилку; $\Psi_{1}$ - относительное изменение температуры уходящих дымовых газов; $\kappa_{1}, \kappa_{2}, \kappa_{3}$ и $\kappa_{4}-$ коэффициенты пропорциональности.

Используя свойство суперпозиций, видим, что все уравнения одинажовые, свойственны инерционному объекту

$$
T \dot{\varphi}+\varrho \varphi=\mu
$$

11 отличаются только числовыми значениями величин.

Определить числовые значения можно либо путем теоретического расчета, либо экспериментально.

Для экспериментального определения преобразуем уравнение (1) и введем в него коэффициент, учитывающий влияние производительности (нагрузки) на ннерционный коэффициент:

$$
a=\frac{G}{G_{\max }},
$$

тде $G$ - текущая производительность установки (расход сланца, поступающего в реактор в единицу времени) и $G_{\max }$ - ее максимальная производительность.

Тогда вместо уравнения (1) будем иметь

$$
\mu-a_{\varrho} \varphi=T \dot{\varphi}
$$

решением которого, как известно, будет

$$
\varphi=\frac{\mu}{a \rho}\left(1-e^{-\frac{a_{\rho}}{T} t}\right)
$$

Величины $a$, е и $\mu$, входящие в уравнение (2), определяются экспериментально, а коэффициент уравнения $T$ вычисляется по этим данным методом наименьших квадратов.

Из опыта получается ряд значений двух неслучайных величин, которые связаны между собой определенной функцией, причем каждому значению независимого переменного соответствует одно значение зависимого переменного во время опыта.

При представлении опытных данных уравнениями требуется подобрать формулу, которая наиболее вероятно представляла бы искомую функциональную зависимость. Форма уравнения известна, и на основании опытных данных необходимо определить значение коэффициентов этих уравнений. 
Приведем уравненне (2) к линейному виду. Имеем

$$
\begin{array}{r}
\frac{q a_{\varrho}}{\mu}=1-e^{-\frac{a_{\rho}}{T} t}, \quad 1-\frac{\varphi a_{\varrho}}{\mu}=e^{-\frac{a \rho}{T} t}, \\
-\frac{2,3}{a \varrho} \log \left(1-\frac{\varphi a_{\varrho}}{\mu}\right)=\frac{1}{T} t . \\
-\frac{2,3}{a_{\varrho}} \log \left(1-\frac{\varphi a_{\varrho}}{\mu}\right)=y .
\end{array}
$$

Обозначаем

Тогда получим окончательно

$$
y=\frac{1}{T} t
$$

Уравнение (3) представляет прямую, общий вид которой, как нзвестно,

$$
z=c+b x
$$

и, следовательно, определению подлежали бы коэффициенты $c$ и $b$.

Однако, если начать измерение параметра после приложения возмущения от равновесного первоначального значения, то коэффициент $c=0$ и опрелелению подлежит только коэффициент $b$. Если наблюдение начинается не с номинального значения параметра, то перенесе ние прямой в начало координат не меняет значения $b$, которое в данном случае нас только и интересует.

Для определения значения $b$ методом наименьших квадратов принимается, что все наблюдения одинакового веса и что ошибкам измерения подвержена лишь переменная $\varphi$.

Коэффициент $T$ определяем из уравнения

$$
T=\frac{\Sigma t^{2}}{\Sigma t y^{\prime}} \text {, }
$$

где $t-$ время.

Проверка правильности найденных значений коэффициентов формул характеризуется коэффициентом средней квадратической ошибки $\sigma_{y}$

$$
\sigma_{y}==\sqrt{\frac{\Sigma(4 y)^{2}}{n}}
$$

где $\Delta y$ - разность значений $y$, полученная путем измерения и определення по найденной формуле; $n$ - число измерений.

Вероятность каждого значения $\varphi$ от $t$ характеризуется коэффищиентом корреляции $r$,

$$
r=\sqrt{\frac{(\Sigma t y)^{2}}{\Sigma t^{2} \Sigma y^{2}}}
$$


На основании нескольких опытов таким методом расчета найдено, что $T=300-580$ сек, $\varrho=1,85-2,9$ и постоянное времени реактора $T_{\mathrm{a}}=\frac{T}{\varrho}=162-215$ сек; при этом $\sigma_{y}=0,13-0,27$ и $r=0,78-0,96$.

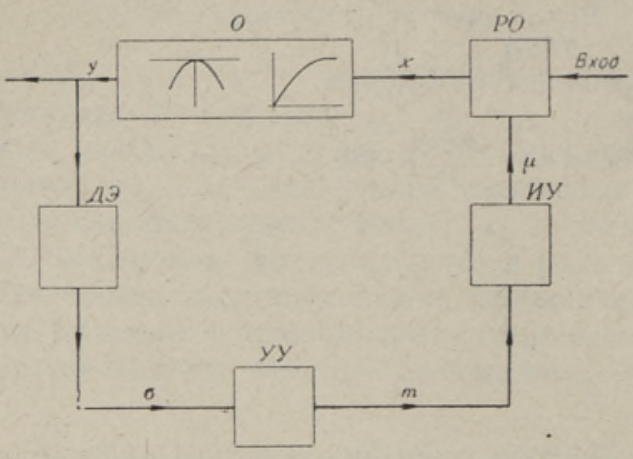

Фиг. 4. Функциональная схема экслремального регулирования: РО - pery. лирующий орган; О - объект регулирования; ДЭ - датчик искания экстремума; УУ - управляющее устройство (экстремальный регулятор); ИУ - исполннтельное устройство.

Исходя из большой инерционности установки и переменностн значения экстремума, из существующих типов экстремального регулирования для данной уста-

новки выбрана релейно-импульсная система с независимым поиском ін реверсированием входящей величины при нулевом значении разностей измерения выходной величины. Входная величина постоянна как по модулю, так и по частоте, а ее знак определяется в зависимости как от предыдущего знака, так и по знаку разности выходной величины. Экстремальный регулятор выбран со ступенчатым изменением входной величины с запоминанием экстремума (фиг. 4). Выходом экстремального регулятора является управляющий сигнал, включающий исполнигельное устройство в необходимом направлении.

Электрическая схема экстремального регулятора прнведена на фиг. 5 и 6. Прнниип работы этого регулятора основан на сравнении импульсов между дискретными равноотстоящими промежутками времени на конденсаторах $\mathrm{C}_{1}$ и $\mathrm{C}_{2}$ (фиг. 5).

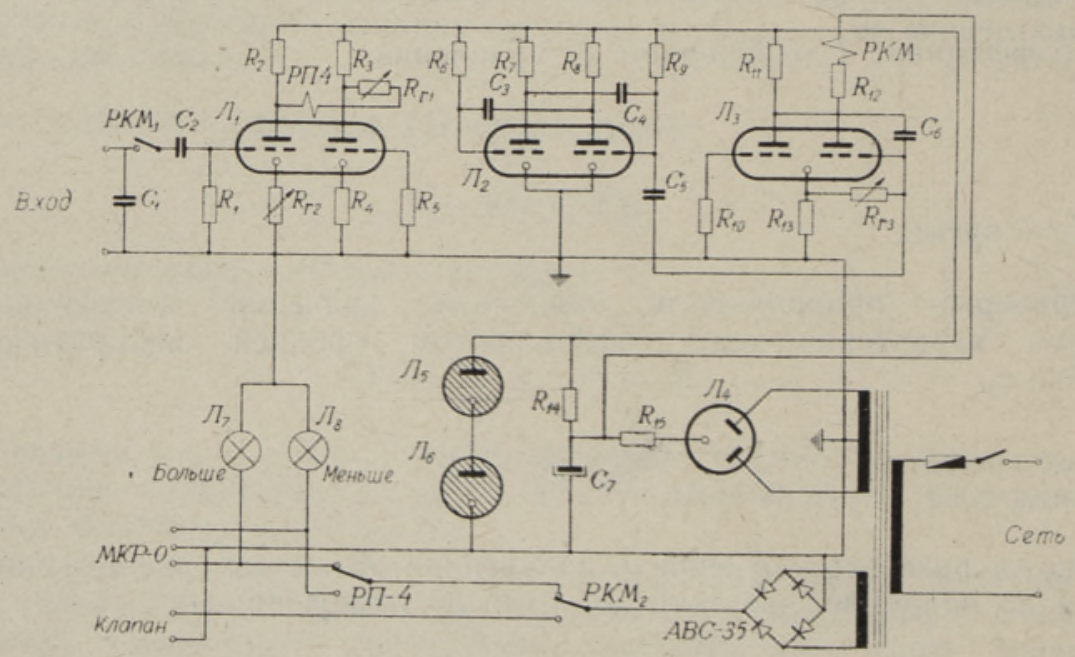

Фнг. 5. Прннципиальная схема экстремального регулятора.

Используется мостовое соединение постоянного тока ( $\mathrm{I}_{1}-6 \mathrm{H1П).} \mathrm{Сравнение} \mathrm{напря-}$ жения на конденсаторах пронзводнтся при помощи мультнвибратора ( $\left.\mathrm{I}_{2}-6 \mathrm{H} 2 \Pi\right)$, нз которого нмпульс направляется в одновибратор $\left(Л_{3}-6 \mathrm{H} 15 \Pi\right)$, который запуска- 
ет управляющее реле РКМ. Последннй имеет контакты РКМ и РК. $\mathrm{PKM}_{1}$ направляют входное напряжение на запоминающий конденсатор $\mathrm{C}_{2}$. После сравнения его с предыдущим входным напряжением на конденсаторе $\mathrm{C}_{1}$ и получення разности этих напряжений последняя подается на одну ветвь моста.

Правая сторона моста $\left(\Omega_{1}\right)$ имеет постоянный анодный ток, на левой половине анодный ток зависит от полярности разностей зарядов на конденсаторах $\mathrm{C}_{1}$ и $\mathrm{C}_{2}$.

Для реверсирования сервомотора при равных зарядах на конденсаторах $\mathrm{C}_{1} \mathrm{H}_{2}$ в мост постоянного тока поставлено реле РП-5, которое управляет работой двух вспомогательных реле типа МРЦ (обмотки $\mathrm{P}_{1}$ и $\mathrm{P}_{2}$ ), и запоминающее реле РП-4 (фиг. 6). В зависимости от полярности импульсов реле РП-5 включает либо реле $\mathrm{P}_{1}$, либо $\mathrm{P}_{2}$. Последние имеют контакты $\mathrm{P}_{11}, \mathrm{P}_{12}, \mathrm{P}_{13}$ и $\mathrm{P}_{21}, \mathrm{P}_{22}, \mathrm{P}_{23}$. Реле РП-4 следит за работой реле РП-5 и включает свои контакты противоположно контактам $\mathrm{P}_{12}$ и $\mathrm{P}_{22}$. При наличии импульса на входе (во время сравнивания потенциалов в конденсатоpax и работы сервомотора) срабатывают реле $\mathrm{P}_{1}$ и $\mathrm{P}_{2}$, которые и направляют работу сервомотора и своими контактами $\mathrm{P}_{13}$ и $\mathrm{P}_{23}$ разъединяют цепь реле РП-4. В этом случае, если потенциалы в конденсаторах $\mathrm{C}_{1}$ и $\mathrm{C}_{2}$ одинаковы, реле РП-5 бездействует. Поскольку реле РП-4 работает в направлении, противоположном реле РП-5, то во время работы серзомотора управляющий ток от выпрямителя проходит через контакты $\mathrm{P}_{23}$ и $\mathrm{P}_{13}$ и контакты РП-4 в магнитный пускатель МКР-0. В результате жтого при нулевом импульсе сервомотор реверсируется, что, как известно, улучшает процесс понска. Қонтакты $\mathrm{PKM}_{2}$ предназначены для открытия и закрытия клапана измерительной трубкн, которая во время срабатывання сервомотора поддерживается открытой. Лампочкн $\Omega_{7}$ и $\mathrm{I}_{5}$ снгнализируют о направлении вращення сервомотора. Для выборя пернода работы регулятора пернод мультивнбратора выбран продолжительностью в 150, 240 и 320 сек. Остальная часть схемы фнг. 6 совпадает со схемой фиг. 5 .

Фнг. 6. Принципиальная схема МКР-О реверснрования сервомотора при. нулевом измерении.

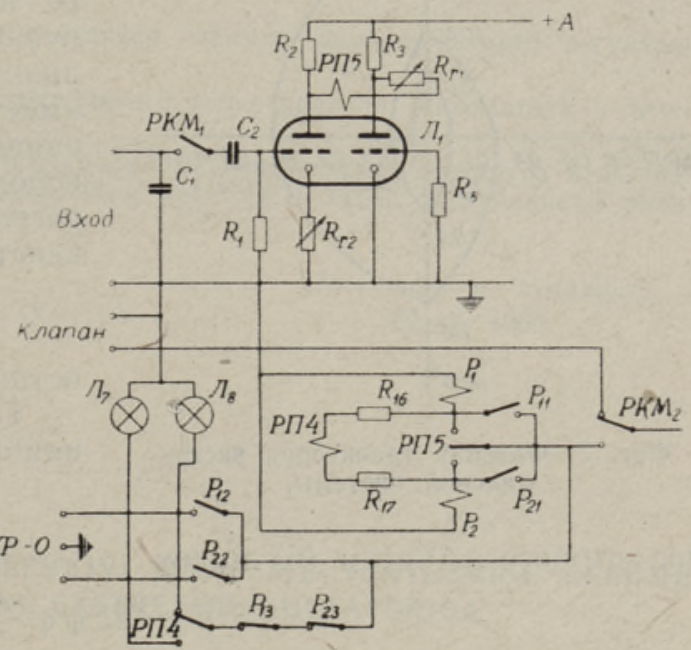

Важную роль при расчете системы экстремального регулирования играют помехи. Для получения правильного импульса регулирования пеобходнмо выбрать частоту регулирования значнтельно меньшую, чем частота случайных функций в виде шума. Чем больше эта разница, тем меньшее влияние оказывают помехи на закон регулирования. Интегрирующим звеном в данной установке является измерительная труба. Она усредняет сигнал помехи и предохраняет систему от ложных двнжений, если периоды работы регулятора выбраны достаточной продолжительности.

Как обычно принято в теоретических расчетах автоматического регулирования, считаем и здесь, что внешнее возмущение малое.

Уравнение кривой, которая имеет максимум, представим в виде уравнения квадратичной функции. Объект представим как последовательно соединенное линейное инерционное и нелинейное безынерционное звенья [1-3].

Уравнение линейной части объекта

$$
T_{a} \dot{\varphi}+\varphi=\kappa \mu \text {. }
$$


Уравнение сервомотора $T_{\mathrm{c}} \dot{\mu}=\sigma u$, где $\sigma$ - время включения сервомотора.

Отсюда находим

$$
\dot{\mu}=\alpha_{1} u,
$$

где $\alpha_{1}=\frac{\sigma}{T_{\mathrm{c}}}$.

Vравнение нелинейной экстремальной характернстики

$$
\sigma=-c \varphi^{2} .
$$

Уравнение измерительного элемента

$$
u=\operatorname{sign}(\dot{\sigma}+\Delta) \operatorname{sign} \dot{\varphi} .
$$

Здесь $\Delta-$ нечувствительность поляризованного реле оптимизатора.

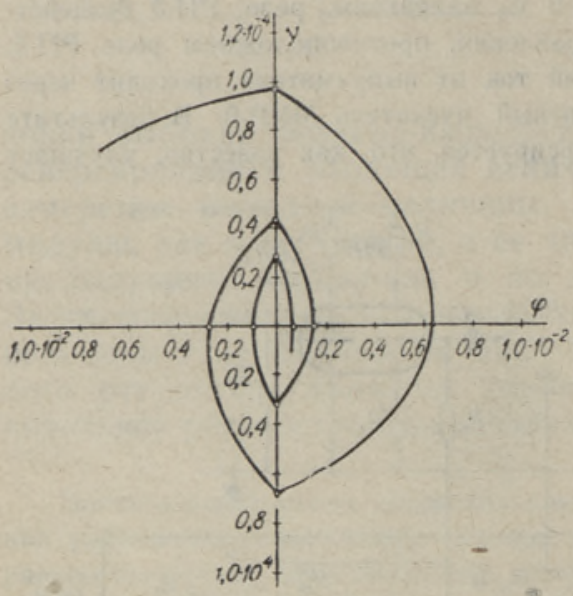

Фиг. 7. Фазовая траекторгя экстремальной системы.

из которого с учетом (6) имеем

Поскольку непосредственное измерение выходного сигнала инерционной части объекта и передача его в логический элемент затруднительно, в экстремальном регуляторе используется сигнал от регулирующего органа. Разница состоит лишь в том, что в последнем случае имеет место некоторое запаздывание изменения температуры вследствие изменения положения регулирующего органа, причем равенство

$$
\operatorname{sign} \dot{\mu}=\operatorname{sign} \dot{\varphi}
$$

остается в силе.

Таким образом, вместо уравнения (7) рассматривается уравнение

$$
u=\operatorname{sign}(\dot{\sigma}+\Delta) \operatorname{sign} \dot{\varphi},
$$

$$
u=-\operatorname{sign}(2 c \varphi \dot{\varphi}-\Delta) \operatorname{sign} \dot{\varphi} .
$$

Поскольку управляющий сигнал $u$ может иметь только значение \pm 1 , то, исключая из уравнения (4) и (5) переменную $\mu$ и учитывая, что нечувствительность регулятора $\Delta$ по сравнению с входным сигналом в регулятор величина ничтожно малая $(\Delta= \pm 0,3-0,5$ в, а диапазон измерения 200 в), получаем уравнение динамики системы (при $\Delta=0)$.

$$
T_{\mathrm{a}} \ddot{\varphi}+\dot{\varphi}+\kappa \alpha_{1} \operatorname{sign} u=0 .
$$

Обозначая $\kappa \alpha_{1}=a$, имеем

$$
T_{\mathrm{a}} \ddot{\varphi}+\dot{\varphi} \pm a=0 .
$$

Решая это уравнение, получим

$$
\varphi=\varphi_{0}+T_{\mathrm{a}}\left(\dot{\varphi}_{0}-\dot{\varphi}\right) \pm a T_{\mathrm{a}} \ln \left(\frac{\dot{\varphi} \pm a}{\dot{\varphi}_{0} \pm a}\right),
$$

где знак «+» действителен для случая $\varphi>0$ и знак «- - для случая $\varphi<0$. 
Фазовая траектория экстремальной системы, т. е. графическое изображение уравнения (13), представлено на фиг. 7 при числовых значениях $T_{\mathrm{a}}=200$ сек, $T_{\mathrm{c}}=2700$ сек и $a=0,275 \cdot \frac{1}{90} \cdot \frac{10}{300}=0,000162$ (т. е. работа сервомотора происходит в течение 10 сек и выдержка в течение 300 сек). чена.

Как видно из этого графика, устойчивость регулирования обеспе-

\title{
Выводы
}

1. Установка термической переработки сланца с твердым теплоносителем является объектом, хорошо поддающимся регулированию.

2. Выход смолы и газа имеет экстремальное значение, зависяшее от температуры смеси теплоносителя и сланца в реакторе.

3. Разработана схема автоматического управления установки с использованием электронного экстремального регулятора.

\section{Л ИТЕРАТУ Р А}

1. А. Г. Нв ахнен ко, Самонастранвающиеся системы автоматического регулирования, Изд. АН УССР, 1955.

2. И. С. Моросанов, Методы экстремального регулирования, Автоматика и телемеханика, № 11, 1957.

3. О. М. Крыж анов ский, В М. Кунце вич, О переходных процессах в экстре мальных снстемах регулировання, Нзв. АН СССР, ОТН, Энергетнка и автоматика, № 3, 1959.
Институт кибернетики
Академии маук Эстонской ССР
Поступнла в редакцию
22. X 1960

\section{TAHKE SOOJUSEKANDJA ABIL POLEVKIVI TERMILISE TOOTLEMISE SEADME AUTOMAATJUHTIMINE EKSTREEMREGULAATORIGA}

\author{
E. Künnap, \\ tehniliste teaduste kandidaat
}

Resümee

Tööstuses kasutusel olevad põlevkivi termilise töötlemise seadmed, nagu tunnelahjud, vertikaalgeneraatorid ja pöörlevad retordid, kasutavad soojusekandjana gaasi ja toorainena tükkpõlevkivi. Tahke soojusekandjaga seadmes on soojusekandjaks tuhk, tooraineks aga peenpõlevkivi. Parema soojusülekande tōttu soojusekandja ja tooraine vahel on viimatí nimetatud seadmete tööprotsess intensiivsem ja nad on paremini reguleeritavad kui esimesed. Peale selle vôivad nende peamisteks ümbertöötamisproduktideks olla nii ôli kuí ka gaas. Artiklis käsitletakse esimest juhtu.

Sellel tööprotsessil on reguleeritaval parameetril (öli hulgal) olemas ekstreemne sōltuvus tuha hulga järgi. Seetōttu on seadme automaatjuhtimiseks otstarbekohane kasutada ekstreemregulaatorit. Instituudis valmistatud elektronekstreemregulaator on automaatjuhtimissüsteemi pearegulaatoriks. Reaktori katseliselt ja analüütiliselt kindlaksmääratud ajakonstandi puhul ja arvutuste pōhjal vałitud regulaatori parameetrite juures on väljaarvutatud reguleerimissüsteem stabiilne.

Eesti NSV Teaduste Akadeemia Küberneetika Instituut
Saabus toimetusse 22. X 1960 


\section{AUTOMATIC CONTROL, WITH AN EXTREME-REGULATOR, OF THE EQUIPMENT FOR A THERMAL TREATMENT OF OIL SHALE WITH A SOLID HEAT-CARRIER}

\section{E. Künnap}

\section{Summary}

The usual industrial equipment for the thermal treatment of oil shale as tunnel ovens, vertical generators and revolving retorts are operated with a heat-carrier - gas and, as raw material, oil shale in pieces. The equipment working with a solid heat-carrier makes use, as a heat-carrier, of ash and, as raw-material - of fine oil shale. Owing to a better transfer of heat from the heat-carrier to the raw material, the working process of the last-mentioned equipment is more intensive and this equipment can be better controlled than the first-mentioned one. Moreover, this equipment may be used for working up oil as well as gas. The first case is examined in the present paper.

In this process, the parameter to be regulated (the amount of oil) has an extreme depending on the amount of ash. Therefore, for an automatic control of the equipment. it is advisable to use an extreme-regulator. The electronic extreme-regulator constructed at the institute is used as main regulator of the automatic control system.

According to the experimentally and analytically determined time constant of the reactor as well as analytically computed parameters of the regulators, the obtained control system is stable.

Academy of Sciences of the Estonian S.S.R., Received Institute of Cybernetics 